\title{
Beneficial effects of catechin-rich green tea and inulin on the body composition of overweight adults
}

\author{
Hsin-Yi Yang ${ }^{1,2}$, Suh-Ching Yang ${ }^{3}$, Jane C.-J. Chao ${ }^{3}$ and Jiun-Rong Chen ${ }^{2,3 *}$ \\ ${ }^{1}$ Department of Nutrition, I-Shou University, Yanchao District, Kaohsiung City, Taiwan, ROC \\ ${ }^{2}$ Nutrition Research Center, Taipei Medical University Hospital, Taipei, Taiwan, ROC \\ ${ }^{3}$ Department of Nutrition and Health Sciences, Taipei Medical University, 250 Wu-Hsin Street, Taipei 110, Taiwan, ROC \\ (Received 8 December 2010 - Revised 16 December 2010 - Accepted 9 February 2011 - First published online 28 October 2011)
}

\section{Abstract}

Green tea catechin has been proposed to have an anti-obesity effect. The aim of the present study was to investigate whether the effect of catechin-rich green tea in combination with inulin affects body weight and fat mass in obese and overweight adults. A total of thirty subjects were divided into a control group and an experimental group who received $650 \mathrm{ml}$ tea or catechin-rich green tea plus inulin. A reduction of body weight $(-1.29($ SEM 0.35$) \mathrm{kg})$ and fat mass $(0.82($ SEM 0.27$) \mathrm{kg})$ in the experimental group was found after 6 weeks, and no adverse effects were observed. After refraining from consumption for 2 weeks, sustained effects on body weight and fat mass were observed. We conclude that continuous intake of catechin-rich green tea in combination with inulin for at least 3 weeks may be beneficial for weight management.

\section{Key words: Green tea: Catechins: Inulin: Obesity: Body fat}

Morbidity from overweight and obesity is increasing, leading to numerous health problems. Prolonged excess energy intake and decreased energy expenditure lead to body fat accumulation. Obesity, especially visceral obesity, carries a strong risk of some related CVD and metabolic diseases, such as the metabolic syndrome, hyperlipidaemia, hypertension and diabetes mellitus ${ }^{(1)}$. Lifestyle modification, especially changing dietary habits, plays an important role in the management of obesity. Both increasing energy expenditure and decreasing energy intake may stimulate weight loss and improvement of body composition.

Green tea is a popular and widely consumed beverage around the world. Green tea-derived polyphenols such as epigallocatechin gallate (EGCG), the most abundant catechin in tea, have been reported to produce physiological effects, including antioxidative, anticancer, anti-obesity and hypolipidaemic effects. Epidemiological studies in Taiwan show an inverse relationship between habitual tea consumption and body fat distribution ${ }^{(2,3)}$. Human and animal studies have explored the mechanism by which green tea catechins may be beneficial for the prevention or treatment of obesity. EGCG may decrease energy intake by reducing food intake, interrupting lipid emulsification and absorption, and increasing energy expenditure via thermogenesis, fat oxidation and faecal lipid excretion, and this so far only has been shown in animal studies. However, information from human studies is still limited ${ }^{(4)}$.

Inulin-type fructans are a linear polydisperse carbohydrate material consisting mainly of $\beta$ - $(2 \leftarrow 1)$ fructosyl-fructose linkages. They resist hydrolysis by human digestive enzymes; thus, they are recognised as a kind of non-digestive oligosaccharide $^{(5)}$. Inulins are now widely used as an ingredient of functional foods for its properties as dietary fibre and prebiotics. A series of studies has demonstrated that inulin affects lipid homeostasis and metabolism, and body weight, though the mechanisms remain unclear ${ }^{(6,7)}$. Therefore, the present study was designed to investigate whether habitual consumption of green tea beverages containing high doses of catechins in combination with inulin can produce weight loss and fat redistribution in overweight subjects.

\section{Materials and methods}

Preparation of green tea

For the experimental group, $28 \mathrm{~g}$ of green tea leaves (Camellia sinensis) were extracted with 2 litres of hot water $\left(70^{\circ} \mathrm{C}\right)$ for $8 \mathrm{~min}$ and then filtered to get the tea extract; for the control group, $8.5 \mathrm{~g}$ of tea leaves were extracted in the same manner as above. In addition, $18 \mathrm{~g}$ inulin powder/1 (>85\%

Abbreviation: EGCG, epigallocatechin gallate.

*Corresponding author: J.-R. Chen, fax +88622737 3112, email syunei@tmu.edu.tw 
fructo-oligosaccharides; average chain length: seven monomers; Sensus, Roosendaal, Netherlands) were added to the experimental green tea as a source of dietary fibre and to make the two beverages indistinguishable in taste. The final catechin concentrations were 81 and $267 \mathrm{mg}$ /bottle in the control and experimental tea, respectively. The two beverages were packed identically in $325 \mathrm{ml}$ bottles.

\section{Subjects}

A total of thirty volunteers, aged 20-50 years old, with BMI $\geq 24 \mathrm{~kg} / \mathrm{m}^{2}$ were recruited for the present trial (Table 1). Subjects were recruited by announcement at Taipei Medical University and Taipei Medical University Hospital (Taipei, Taiwan). The exclusion criteria were as follows: use of medications and supplements; consumption of other tea products 1 week before and during the experimental period; postmenopausal women; systemic, liver, renal and digestive diseases. Before the experiment, we explained the content, purpose and possible risks of the study to all subjects and written informed consent was obtained from all subjects.

\section{Study design}

The study was conducted according to the guidelines laid down in the Declaration of Helsinki and all procedures involving human subjects/patients were approved by the Human Investigation Review Board of Taipei Medical University and performed under the supervision of a family medicine doctor and dietitians from the Taipei Medical University Hospitals. Written informed consent was obtained from all subjects. Subjects were assigned to either the control ( $n$ 15) or the experimental group ( $n$ 15) through stratified randomisation according to age, sex and BMI. During the 6-week experimental period, subjects ingested the tea beverage $(650 \mathrm{ml} / \mathrm{d})$ and consumed three meals $(7531-8368 \mathrm{~kJ})$ per $\mathrm{d}$ offered by the Taipei Medical University Hospital. The consumption of other food and beverages containing catechins, polyphenols or caffeine was prohibited. Subjects were also advised to maintain their physical activity levels. After the 6-week experimental period, all subjects continued with another 2-week follow-up period without ingestion of any tea beverages. Subjects were asked to record their daily dietary intake for $3 \mathrm{~d}$ at baseline, 6th and 8th weeks, and the records were reviewed by a dietitian.

\section{Anthropometric measurements}

Height, body weight, waist circumference and hip circumference were measured while the subjects were in standing position at baseline, 3rd, 6th and 8th weeks. Waist circumference was assessed using a tape measure around the mid-distance between the last rib margin and the iliac crest. Hip circumference was measured at the level of the largest circumference around the hip. The body fat ratio was measured by the bioimpedance analysis method (InBody 3.0 body composition analyser; Biospace, Seoul, Korea).

\section{Blood pressure measurements}

After a 5 min rest, blood pressure was measured on the right arm with an automatic blood pressure monitor (FT-500R; Jawon, Kyungsan, Korea). Systolic and diastolic blood pressure was calculated as the average of three measurements.

\section{Blood sampling and clinical analysis}

Blood samples were collected at baseline, 3rd, 6th and 8th weeks of the experiment. Subjects fasted for $12 \mathrm{~h}$ before blood sampling. All biochemical analyses were carried out on a Hitachi 7170 Autoanalyser (Hitachi, Tokyo, Japan). We also measured cardiopulmonary functions of all subjects at the beginning and end of the study. The electrocardiogram was recorded with the Page Writer Trim III Cardiograph (Philips, Burlington, MA, USA) and the lung function test was performed with a spirometer (KoKo Legend Spirometer; nSpire Health, Longmont, CO, USA).

\section{Statistical analysis}

All evaluation data are presented as means with their standard errors. The difference in percentage of males and females between the control and experimental groups was assessed

Table 1. Characteristics of the subjects before and after the intervention (Mean values with their standard errors, $n$ 15)

\begin{tabular}{|c|c|c|c|c|c|c|c|c|c|c|c|c|}
\hline & \multicolumn{6}{|c|}{ Control group } & \multicolumn{6}{|c|}{ Experimental group } \\
\hline & \multicolumn{2}{|c|}{ Wo } & \multicolumn{2}{|c|}{ W6 } & \multicolumn{2}{|c|}{ W8 } & \multicolumn{2}{|c|}{ wo } & \multicolumn{2}{|c|}{ W6 } & \multicolumn{2}{|c|}{ W8 } \\
\hline & Mean & SEM & Mean & SEM & Mean & SEM & Mean & SEM & Mean & SEM & Mean & SEM \\
\hline Age (years) & $25 \cdot 5$ & 1.5 & & & & & $27 \cdot 6$ & $2 \cdot 1$ & & & & \\
\hline Sex (male/female) & \multicolumn{6}{|c|}{$8 / 7$} & \multicolumn{6}{|c|}{$8 / 7$} \\
\hline Weight $(\mathrm{kg})$ & $73 \cdot 2$ & $2 \cdot 6$ & $73 \cdot 1$ & $2 \cdot 6$ & $73 \cdot 3$ & $2 \cdot 8$ & $76 \cdot 7$ & $3 \cdot 1$ & $74.7^{*}$ & $3 \cdot 1$ & $74 \cdot 9^{*}$ & $3 \cdot 2$ \\
\hline $\mathrm{BMI}\left(\mathrm{kg} / \mathrm{cm}^{2}\right)$ & $26 \cdot 7$ & 0.7 & $26 \cdot 7$ & 0.7 & $26 \cdot 8$ & 0.7 & $27 \cdot 3$ & 0.9 & $26 \cdot 6^{*}$ & 0.9 & $26 \cdot 7^{\star}$ & 0.9 \\
\hline Waist circumference $(\mathrm{cm})$ & $87 \cdot 2$ & $2 \cdot 0$ & $88 \cdot 1$ & $2 \cdot 0$ & $87 \cdot 2$ & $2 \cdot 1$ & $89 \cdot 8$ & $2 \cdot 3$ & $88 \cdot 3$ & $2 \cdot 5$ & $87 \cdot 8^{*}$ & $2 \cdot 5$ \\
\hline Hip circumference $(\mathrm{cm})$ & $105 \cdot 3$ & 1.6 & $105 \cdot 0$ & 1.5 & $104 \cdot 5^{\star}$ & $1 \cdot 6$ & $107 \cdot 1$ & 1.8 & $105 \cdot 2^{*}$ & 1.9 & $104 \cdot 5^{\star}$ & 1.9 \\
\hline Total fat mass $(\mathrm{kg})$ & $23 \cdot 2$ & $1 \cdot 2$ & $22 \cdot 7$ & $1 \cdot 2$ & $22 \cdot 9$ & 1.2 & $24 \cdot 8$ & 1.7 & $23 \cdot 1^{*}$ & 1.8 & $23 \cdot 4^{*}$ & 1.7 \\
\hline Lean body mass (kg) & $45 \cdot 9$ & 1.6 & $46 \cdot 2$ & $1 \cdot 7$ & $46 \cdot 3$ & $1 \cdot 7$ & 47.5 & 1.9 & $47 \cdot 4^{*}$ & 1.9 & $47 \cdot 2$ & $2 \cdot 0$ \\
\hline
\end{tabular}

W, week.

${ }^{*}$ Mean values were significantly different from W0 in the same group $(P<0.05)$. 
by the $\chi^{2}$ test. The age, body composition, anthropometric and biochemical analysis of the two groups were evaluated by Student's $t$ test, and differences between the values of each time point and the initial measurement were analysed by the paired $t$ test. A $P$ value $<0.05$ was considered to be statistically significant.

\section{Results}

\section{Subjects and dietary intake}

Basal characteristics of the subjects assigned to different groups are shown in Table 1. No difference was found in age, weight, BMI, fat mass, waist and hip circumference between the control


and experimental groups at baseline. All subjects consumed three meals per $\mathrm{d}$ offered by the hospital and were also asked to keep diet records at baseline, 6th and 8th weeks to evaluate their dietary intake. There was no significant difference in energy intake or protein, fat and carbohydrate composition of the diet between the two groups during the experimental and follow-up periods $(P>0 \cdot 05)$. All subjects consumed their study beverage as instructed during the experimental period.

\section{Body weight, body composition, waist and hip circumference}

Reduction in body weight, BMI and fat mass in the experimental group was significantly different from that of the control
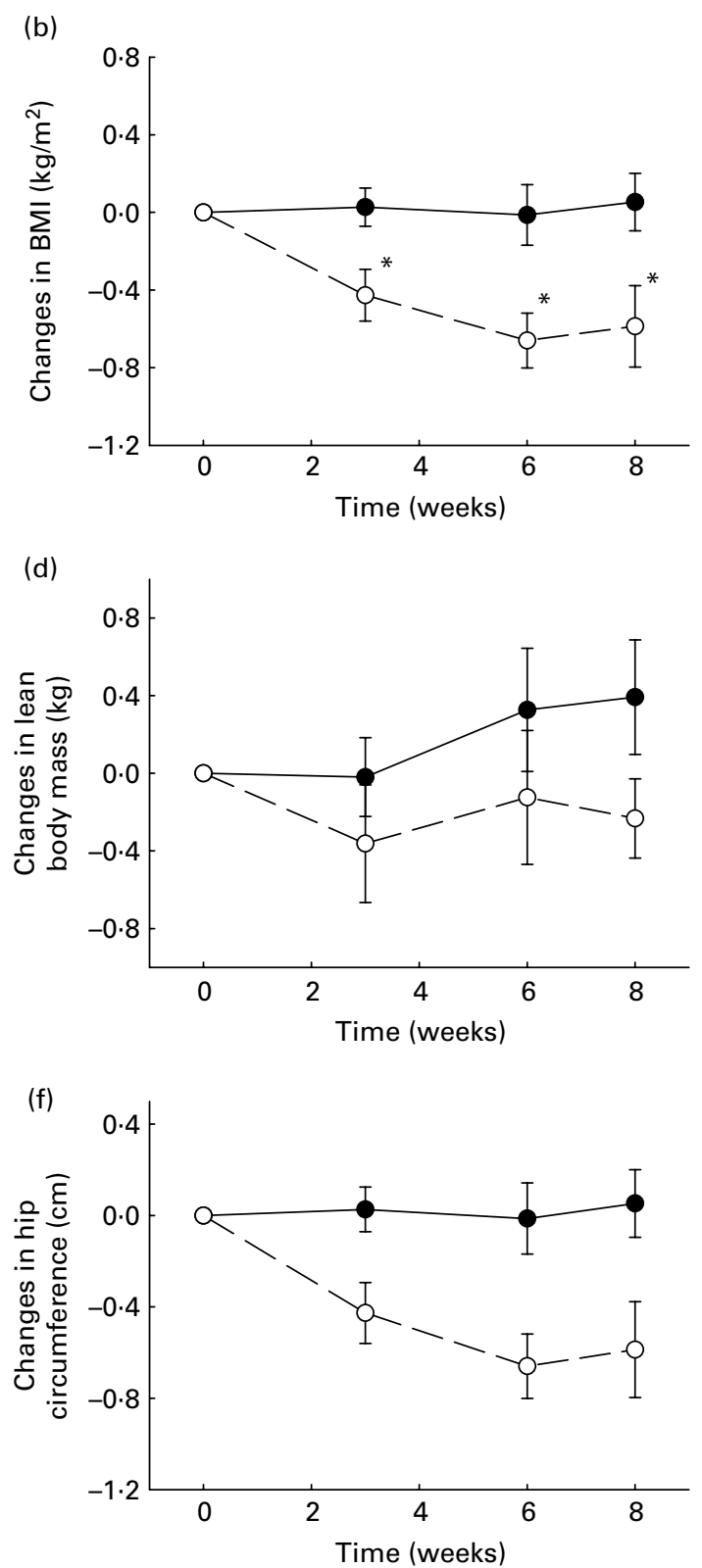

Fig. 1. Changes in (a) weight, (b) BMI, (c) fat mass, (d) lean body mass, (e) waist circumference and (f) hip circumference during the experimental and follow-up periods. Values are means, with their standard errors represented by vertical bars $(n 15)$. * Mean values were significantly different from those of the control group $(P<0.05 ;$ Student's $t$ test $)$. 
group at 3rd and 6th weeks, and differences were maintained at the 2-week follow-up period, as shown in Fig. 1. Hip and waist circumference was reduced in the experimental group compared with the control group after consuming the study beverage for 6 weeks, although no significant difference was found (waist, $P=0.07$; hip, $P=0.06$ ).

\section{Blood pressure and blood biochemical analysis}

Blood pressure and blood chemical measurement of all subjects were within the normal range during the experimental period. After consuming the study beverage for 6 weeks, blood pressure and blood glucose concentrations were significantly decreased in the experimental group. Diastolic blood pressure and blood glucose in the control group were also lower than the baseline values. Blood lipids in both groups showed no change during the experimental and follow-up periods (Table 2).

\section{Safety and tolerability}

No adverse event was found in either group. Hepatic and renal function parameters were all within the normal range (Table 2), and cardiopulmonary function and electrocardiograms were normal in all subjects during the experimental and follow-up periods.

\section{Discussion}

The present study shows that daily consumption of $650 \mathrm{ml}$ catechin-rich green tea beverage plus inulin $(534 \mathrm{mg}$ catechins and $11.7 \mathrm{~g}$ inulin) was acceptable and no adverse effect was observed in our subjects. After 6 weeks, body weight, BMI, fat mass and blood pressure were significantly decreased in the experimental group. The result shows that catechin-rich green tea plus inulin may have beneficial effects in people who are overweight.

For overweight patients, reducing weight and fat mass is the main target of diet modification. Many studies have reported the effects of tea catechins on lipid metabolism and body fat accumulation. Long-term consumption of tea catechins helps to suppress high-fat diet-induced obesity via activation of hepatic lipid metabolism ${ }^{(8)}$. Daily intake of $300 \mathrm{mg}$ EGCG, the main catechin in green tea, also has the potential to increase fat oxidation in men ${ }^{(9)}$. In addition, tea catechins may reduce the deposition of visceral fat and hepatic fatty acid synthesis ${ }^{(10)}$, and EGCG supplementation prevents obesity by reducing adipose tissue mass by inhibiting adipocyte differentiation and reducing lipogenic enzyme expression ${ }^{(11)}$. The present study found that catechin-rich green tea decreased fat mass and BMI. Although another study has found that administration of tea catechins during a lowenergy diet had no additional effect on body weight and body composition, and speculated that this may be due to increased hunger with green tea treatment ${ }^{(12)}$, we found no difference in energy intake between the control and experimental groups. Weight loss associated with green tea catechins may involve its thermogenic properties and promoting fat oxidation $^{(13)}$. Green tea may suppress body fat by reducing digestibility of foods and increasing brown adipose tissue thermogenesis via $\beta$-adrenoceptor activation ${ }^{(14)}$. In one animal study, long-term EGCG treatment $(3 \cdot 2 \mathrm{~g} / \mathrm{kg})$ attenuated the development of obesity and visceral fat ${ }^{(15)}$. In a human study, daily consumption of tea containing $690 \mathrm{mg}$ catechins for 12 weeks reduced body fat in healthy Japanese men ${ }^{(16)}$, and catechin-rich beverages $(576 \mathrm{mg}$ ) ameliorated obesity and decreased waist circumference and systolic blood pressure when compared with original green tea ( $75 \mathrm{mg}$ catechins) without raising safety concerns in Japanese children ${ }^{(17)}$. However, a double-blind study has found no effects of green tea catechins on weight management ${ }^{(12)}$. Another study has demonstrated that catechin-rich tea extract $(583 \mathrm{mg} / \mathrm{d})$ led to a reduction in body fat in Japanese women and men with visceral fat-type obesity after a 12 -week experiment ${ }^{(18)}$. In addition, inulin-type fructan can also reduce body-weight gain $^{(7)}$. In the present study, we added $18 \mathrm{~g}$ inulin/ 1 to a catechin-rich green tea beverage and observed significant weight loss and fat mass reduction after 3 weeks, when compared with the control group, and the effects remained 2 weeks after refraining from consumption. These results suggest that inulin and green tea catechins in our beverage may be the effective components to induce weight loss and fat mass. However, the mechanisms by which inulin improves weight management is still very limited and needs further investigation.

Green tea also contains caffeine, which may stimulate thermogenesis, and $150 \mathrm{mg}$ caffeine/600 mg catechins correspond to approximately the content in three cups of green tea ${ }^{(19)}$. A meta-analysis has also shown that caffeine intake may influence the effect of catechins on weight loss and weight maintenance ${ }^{(20)}$. Habitual high caffeine intake was associated with a greater weight loss and relatively higher thermogenesis and fat oxidation. However, this association has been found during the weight-loss phase when subjects were put on a low-energy diet ${ }^{(21)}$. In a clinical study, subjects treated with caffeine in amounts equivalent to those found in green tea extract also showed no effect on energy expenditure and respiratory quotient ${ }^{(13)}$. Thus, the effect of catechin-rich green tea beverage on body weight and fat mass cannot be completely explained by its caffeine content.

A study has reported that green tea consumption protects the cardiovascular system ${ }^{(18)}$. In the present study, neither the control group nor the experimental group showed a change in blood lipids after the experimental period. A crosssectional study has demonstrated an inverse association between green tea consumption and serum lipids ${ }^{(22)}$. Another study has also shown that habitual intake of green tea produced a significant reduction in plasma cholesterol and $\mathrm{TAG}^{(23)}$. Inulin intake may also regulate lipid homeostasis by decreasing fatty acid synthase mRNA in hepatocytes ${ }^{(5)}$. A possible reason for the absence of hypolipidaemic effect found by the present study may be that the baseline blood lipid levels of our subjects were within the normal range. Hypertension is also a CVD risk in obese subjects, and habitual tea drinkers have a lower risk of developing hypertension ${ }^{(24)}$. Consistent with this, we found a decrease in systolic and diastolic 
Table 2. Biochemical analysis and blood pressure of the subjects during the experimental and follow-up periods

(Mean values with their standard errors, $n$ 15)

\begin{tabular}{|c|c|c|c|c|c|c|c|c|c|c|c|c|c|c|c|c|}
\hline & \multicolumn{8}{|c|}{ Control group } & \multicolumn{8}{|c|}{ Experimental group } \\
\hline & \multicolumn{2}{|c|}{ wo } & \multicolumn{2}{|c|}{ W3 } & \multicolumn{2}{|c|}{ W6 } & \multicolumn{2}{|c|}{ W8 } & \multicolumn{2}{|c|}{ wo } & \multicolumn{2}{|c|}{ W3 } & \multicolumn{2}{|c|}{ W6 } & \multicolumn{2}{|c|}{ W8 } \\
\hline & Mean & SEM & Mean & SEM & Mean & SEM & Mean & SEM & Mean & SEM & Mean & SEM & Mean & SEM & Mean & SEM \\
\hline $\mathrm{SBP}(\mathrm{mmHg})$ & $120 \cdot 3$ & 3.2 & $114.5^{\star}$ & 3.3 & $115 \cdot 8$ & 3.6 & $117 \cdot 9$ & 3.7 & 128.7 & 3.4 & $120 \cdot 9^{*}$ & $3 \cdot 1$ & $120 \cdot 7^{\star}$ & 2.5 & $121 \cdot 4^{*}$ & $3 \cdot 6$ \\
\hline $\mathrm{DBP}(\mathrm{mmHg})$ & 73.5 & $2 \cdot 2$ & $68 \cdot 3^{*}$ & $2 \cdot 7$ & $68 \cdot 3^{*}$ & $2 \cdot 1$ & 70.5 & $2 \cdot 2$ & $77 \cdot 3$ & $2 \cdot 4$ & $70 \cdot 9^{*}$ & $2 \cdot 2$ & $71.9^{*}$ & 1.6 & $75 \cdot 4$ & 1.9 \\
\hline Heart rate (times $/ \mathrm{min}$ ) & $77 \cdot 7$ & $3 \cdot 2$ & 73.9 & $2 \cdot 2$ & 74.5 & 2.5 & $75 \cdot 2$ & 1.5 & 81.0 & 3.0 & $75 \cdot 8$ & 3.4 & 76.6 & $2 \cdot 8$ & 79.9 & $2 \cdot 6$ \\
\hline Glucose $(\mathrm{mmol} / \mathrm{l})$ & $5 \cdot 7$ & 0.2 & $5 \cdot 3^{*}$ & 0.2 & $5 \cdot 3^{\star}$ & 0.2 & $5 \cdot 5^{*}$ & 0.2 & 5.5 & 0.2 & $5 \cdot 1^{*}$ & 0.1 & $5 \cdot 2^{*}$ & 0.1 & $5 \cdot 2^{*}$ & 0.1 \\
\hline Albumin $(g / l)$ & 47 & 0 & 36 & 1 & 47 & 1 & 46 & 1 & 47 & 1 & 46 & 1 & 47 & 1 & 47 & 1 \\
\hline BUN (mg/l) & 114 & 6 & 115 & 5 & 110 & 4 & 123 & 9 & 104 & 5 & 109 & 5 & 114 & 6 & 113 & 7 \\
\hline Creatinine $(\mathrm{mg} / \mathrm{l})$ & 8 & 1 & 8 & 1 & 9 & 1 & 9 & 1 & 8 & 1 & 8 & 1 & 8 & 1 & 8 & 1 \\
\hline Uric acid $(\mathrm{mg} / \mathrm{l})$ & 67 & 5 & 64 & 4 & 65 & 5 & 64 & 4 & 61 & 4 & 61 & 4 & 62 & 4 & 61 & 3 \\
\hline Cholesterol $(\mathrm{mmol} / \mathrm{l})$ & 4.8 & 0.2 & 4.9 & 0.3 & 4.7 & 0.3 & 4.8 & 0.2 & 4.6 & 0.6 & 4.4 & 0.3 & 4.4 & 0.6 & $4 \cdot 6$ & 0.2 \\
\hline $\mathrm{TAG}(\mathrm{mmol} / \mathrm{l})$ & $1 \cdot 1$ & 0.2 & 1.3 & 0.2 & 1.1 & 0.2 & 1.2 & 0.2 & 1.0 & 0.1 & 1.0 & 0.1 & 0.9 & 0.1 & $1 \cdot 1$ & 0.1 \\
\hline GOT (U/I) & $31 \cdot 7$ & 8.9 & $24 \cdot 6$ & 4.6 & $26 \cdot 0$ & $6 \cdot 8$ & $24 \cdot 6$ & 4.9 & $20 \cdot 7$ & 1.2 & $19 \cdot 3$ & $1 \cdot 1$ & $20 \cdot 2$ & 1.9 & $26 \cdot 3$ & $6 \cdot 6$ \\
\hline GPT (U/I) & $24 \cdot 3$ & $6 \cdot 3$ & 30.7 & $8 \cdot 7$ & $31 \cdot 7$ & $10 \cdot 8$ & 31.5 & 9.5 & $23 \cdot 1$ & $2 \cdot 9$ & 23.6 & 3.2 & 23.7 & $4 \cdot 1$ & $32 \cdot 1$ & $9 \cdot 6$ \\
\hline$\gamma-\mathrm{GT}(\mathrm{U} / \mathrm{I})$ & $19 \cdot 7$ & $6 \cdot 3$ & $25 \cdot 0$ & $8 \cdot 3$ & $27 \cdot 7$ & 9.5 & $26 \cdot 3$ & 7.9 & 17.9 & 3.0 & $16 \cdot 4$ & $2 \cdot 2$ & $18 \cdot 7$ & $2 \cdot 3$ & $26 \cdot 2$ & $7 \cdot 0$ \\
\hline HDL-C (mg/l) & 533 & 27 & 507 & 29 & 493 & 22 & 481 & 21 & 594 & 45 & 537 & 32 & 542 & 33 & 557 & 33 \\
\hline LDL-C (mg/l) & 1199 & 78 & 1259 & 84 & 1249 & 98 & 1221 & 59 & 1089 & 89 & 1071 & 88 & 1086 & 93 & 1101 & 67 \\
\hline $\mathrm{Na}(\mathrm{mEg} / \mathrm{l})$ & 141.6 & $2 \cdot 1$ & $141 \cdot 2$ & 0.4 & $143 \cdot 2$ & 0.3 & 143.8 & 0.4 & $140 \cdot 4$ & 1.5 & 141.7 & 0.4 & 143.2 & 0.5 & 143.0 & 0.6 \\
\hline $\mathrm{K}(\mathrm{mEq} / \mathrm{l})$ & 4.4 & 0.1 & 4.2 & 0.1 & 4.1 & 0.0 & $4 \cdot 2$ & 0.1 & 4.7 & 0.1 & 4.3 & 0.1 & 4.3 & 0.1 & 4.4 & 0.1 \\
\hline NEFA & 0.5 & $0 \cdot 1$ & 0.5 & 0.1 & 0.4 & 0.0 & 0.5 & 0.0 & 0.5 & 0.1 & 0.5 & 0.0 & 0.4 & 0.0 & 0.5 & $0 \cdot 1$ \\
\hline $\mathrm{T} 4(\mu \mathrm{g} / \mathrm{l})$ & 83 & 3 & 76 & 3 & 80 & 2 & 76 & 3 & 91 & 3 & 86 & 6 & 87 & 6 & 91 & 6 \\
\hline Leptin & $40 \cdot 2$ & $5 \cdot 3$ & 36.6 & 4.3 & 36.9 & 4.8 & 35.7 & 3.9 & $35 \cdot 6$ & 4.7 & 33.8 & 4.8 & $32 \cdot 0$ & 4.6 & 34.0 & 4.0 \\
\hline Erythrocytes $\left(10^{6} / \mu \mathrm{l}\right)$ & 4.7 & 0.1 & 4.7 & 0.1 & 4.7 & 0.1 & 4.7 & 0.1 & 4.7 & 0.1 & 4.7 & 0.1 & 4.7 & 0.1 & $4 \cdot 8$ & $0 \cdot 1$ \\
\hline Leucocytes $\left(10^{3} / \mu \mathrm{l}\right)$ & 7.5 & 0.5 & $7 \cdot 3$ & 0.5 & 6.7 & 0.3 & $7 \cdot 3$ & 0.4 & $6 \cdot 8$ & 0.4 & $6 \cdot 8$ & 0.4 & 6.9 & 0.3 & $7 \cdot 0$ & 0.4 \\
\hline $\mathrm{Hb}(\mathrm{g} / \mathrm{l})$ & 137 & 4 & 137 & 4 & 136 & 4 & 137 & 4 & 144 & 4 & 140 & 4 & 140 & 4 & 142 & 4 \\
\hline Haematocrit (\%) & $40 \cdot 1$ & 1.2 & $40 \cdot 3$ & $1 \cdot 2$ & 40.5 & 1.1 & 40.8 & $1 \cdot 2$ & $41 \cdot 6$ & 1.2 & 40.8 & $1 \cdot 1$ & 41.4 & $1 \cdot 2$ & $42 \cdot 1$ & 1.2 \\
\hline $\operatorname{MCV}(f l)$ & 84.6 & 1.4 & 86.5 & 1.7 & $86 \cdot 8$ & 1.7 & 87.4 & 1.7 & 87.6 & 1.7 & 87.1 & 1.7 & 87.5 & 1.7 & $87 \cdot 8$ & 1.7 \\
\hline
\end{tabular}

W, week; SBP, systolic blood pressure; DBP, diastolic blood pressure; BUN, blood urea nitrogen; GOT, glutamate oxaloacetate transaminase; GPT, glutamate pyruvate transaminase, $\gamma$-GT, $\gamma$-glutamyltransferase; HDL-C, HDL-cholesterol; LDL-C, LDL-cholesterol; T4, thyroxine; MCV, mean corpuscular value.
* Mean values were significantly different from W0 in the same group $(P<0.05 ;$ paired $t$ test) 
blood pressure 3-6 weeks after consuming a catechin-rich beverage plus inulin, and systolic blood pressure remained lower than baseline even after the consumption had been stopped for 2 weeks. These results suggest that catechin-rich green tea beverage may also be beneficial for cardiovascular health.

In conclusion, frequent intake of catechin-rich green tea in combination with inulin for more than 3 weeks may contribute to weight loss, fat mass reduction and lower blood pressure. The beneficial effects of catechin-rich green tea beverage plus inulin suggest that sustained drinking may contribute to managing weight in overweight populations, and prevent further CVD and metabolic diseases.

\section{Acknowledgements}

The present study was funded by the Functional Food Research Fund (Taipei Medical University, Taipei, Taiwan), The contribution of each author was as follows: H.-Y. Y., S.-C. Y. and J. C.-J. C. performed the experiments and contributed to the writing of the manuscript; J.-R. C. designed the study, and was the promoter, investigator and coordinator. All authors read and approved the final contents of the manuscript. The authors declare that there are no conflicts of interest and adhere to the Committee on Publication Ethics guidelines on research and publication practice.

\section{References}

1. Matsuzawa Y (2010) Establishment of a concept of visceral fat syndrome and discovery of adiponectin. Proc Jpn Acad Ser B Phys Biol Sci 86, 131-141.

2. Wu CH, Lu FH, Chang CS, et al. (2003) Relationship among habitual tea consumption, percent body fat, and body fat distribution. Obes Res 11, 1088-1095.

3. Wolfram S, Wang Y \& Thielecke F (2006) Anti-obesity effects of green tea: from bedside to bench. Mol Nutr Food Res $\mathbf{5 0}$, $176-187$

4. Thielecke F \& Boschmann M (2009) The potential role of green tea catechins in the prevention of the metabolic syndrome - a review. Phytochemistry 70, 11-24.

5. Roberfroid MB (2007) Inulin-type fructans: functional food ingredients. J Nutr 137, 2493S-2502S.

6. Delzenne NM, Daubioul C, Neyrinck A, et al. (2002) Inulin and oligofructose modulate lipid metabolism in animals: review of biochemical events and future prospects. $\mathrm{Br} J$ Nutr 87, Suppl. 2, S255-S259.

7. Urias-Silvas JE, Cani PD, Delmee E, et al. (2008) Physiological effects of dietary fructans extracted from Agave tequilana Gto. and Dasylirion spp. Br J Nutr 99, 254-261.

8. Murase T, Nagasawa A, Suzuki J, et al. (2002) Beneficial effects of tea catechins on diet-induced obesity: stimulation of lipid catabolism in the liver. Int J Obes Relat Metab Disord 26, 1459-1464.
9. Boschmann M \& Thielecke F (2007) The effects of epigallocatechin-3-gallate on thermogenesis and fat oxidation in obese men: a pilot study. J Am Coll Nutr 26, 389S-395S.

10. Ikeda I, Hamamoto R, Uzu K, et al. (2005) Dietary gallate esters of tea catechins reduce deposition of visceral fat, hepatic triacylglycerol, and activities of hepatic enzymes related to fatty acid synthesis in rats. Biosci Biotechnol Biochem 69, 1049-1053.

11. Wolfram S, Raederstorff D, Wang Y, et al. (2005) TEAVIGO (epigallocatechin gallate) supplementation prevents obesity in rodents by reducing adipose tissue mass. Ann Nutr Metab 49, 54-63.

12. Diepvens K, Kovacs EM, Nijs IM, et al. (2005) Effect of green tea on resting energy expenditure and substrate oxidation during weight loss in overweight females. Br J Nutr $\mathbf{9 4}$, 1026-1034.

13. Dulloo AG, Duret C, Rohrer D, et al. (1999) Efficacy of a green tea extract rich in catechin polyphenols and caffeine in increasing 24-h energy expenditure and fat oxidation in humans. Am J Clin Nutr 70, 1040-1045.

14. Choo JJ (2003) Green tea reduces body fat accretion caused by high-fat diet in rats through beta-adrenoceptor activation of thermogenesis in brown adipose tissue. J Nutr Biochem 14, 671-676.

15. Bose M, Lambert JD, Ju J, et al. (2008) The major green tea polyphenol, (-)-epigallocatechin-3-gallate, inhibits obesity, metabolic syndrome, and fatty liver disease in high-fat-fed mice. $J$ Nutr 138, 1677-1683.

16. Nagao T, Komine Y, Soga S, et al. (2005) Ingestion of a tea rich in catechins leads to a reduction in body fat and malondialdehyde-modified LDL in men. Am J Clin Nutr 81, $122-129$.

17. Matsuyama T, Tanaka Y, Kamimaki I, et al. (2008) Catechin safely improved higher levels of fatness, blood pressure, and cholesterol in children. Obesity (Silver Spring) 16, $1338-1348$.

18. Nagao T, Hase T \& Tokimitsu I (2007) A green tea extract high in catechins reduces body fat and cardiovascular risks in humans. Obesity (Silver Spring) 15, 1473-1483.

19. Reto M, Figueira ME, Filipe HM, et al. (2007) Chemical composition of green tea (Camellia sinensis) infusions commercialized in Portugal. Plant Foods Hum Nutr 62, 139-144.

20. Hursel R, Viechtbauer W \& Westerterp-Plantenga MS (2009) The effects of green tea on weight loss and weight maintenance: a meta-analysis. Int J Obes (Lond) 33, 956-961.

21. Westerterp-Plantenga MS, Lejeune MP \& Kovacs EM (2005) Body weight loss and weight maintenance in relation to habitual caffeine intake and green tea supplementation. Obes Res 13, 1195-1204.

22. Imai K \& Nakachi K (1995) Cross sectional study of effects of drinking green tea on cardiovascular and liver diseases. BMJ 310, 693-696.

23. Sasazuki S, Kodama H, Yoshimasu K, et al. (2000) Relation between green tea consumption and the severity of coronary atherosclerosis among Japanese men and women. Ann Epidemiol 10, 401-408.

24. Yang YC, Lu FH, Wu JS, et al. (2004) The protective effect of habitual tea consumption on hypertension. Arch Intern Med 164, 1534-1540. 